\title{
Shear wave elastography of the uterine cervix under different conditions with inter-operator agreement analysis
}

\author{
Aysegul Akdogan Gemici ${ }^{A, B, C, D, E, F}$, Aycan Boluk Gulsever ${ }^{B, C, D, E}$, Aysun Fendal Tunca ${ }^{A, C, D, F}$, Elif Hocaoglu ${ }^{A, B, C, D}$, \\ Ercan Inci $\mathrm{A}^{\mathrm{A}, \mathrm{C}, \mathrm{D}, \mathrm{E}, \mathrm{G}}$
}

Bakirkoy Dr. Sadi Konuk Training and Research Hospital, Istanbul, Turkey

\begin{abstract}
Purpose: Shear wave elastography (SWE) is a relatively new technique for measuring tissue elasticity. Its implementation for assessing the tissue of the cervix is evolving, and SWE analyses of healthy, nonpregnant cervixes is the first step in understanding other SWE changes related to cervical pathologies; nevertheless, some challenges in the use of the technique still require investigation. We aimed to target the consistency of healthy cervix shear wave elastography measurements and examine the changes induced by patient-related factors.
\end{abstract}

Material and methods: Elastograms were obtained at the internal and external os in the anterior (IA, EA) and posterior (IP, EP) portions of the cervix using a transvaginal approach in eight postmenopausal and 25 premenopausal women. Measurements with a standard deviation of over $20 \%$ and patients who presented with colour loss or heterogeneity were excluded from the study. Shear wave elastography assessments were performed using a Toshiba Aplio 500 version 6. Statistical significance was defined as a p value less than 0.10 , due to the small number of patients.

Results: The mean speeds obtained at the external os on the anterior and posterior aspects was $3.17 \pm 0.85 \mathrm{~m} / \mathrm{s}$ and 3.18 $\pm 0.84 \mathrm{~m} / \mathrm{s}$, respectively, and at the internal os, the results on the anterior and posterior aspects were $3.38 \pm 0.73 \mathrm{~m} / \mathrm{s}$ and $3.53 \pm 0.81 \mathrm{~m} / \mathrm{s}$, respectively. The difference in speed among all regions was statistically significant $(p<0.05)$. Fifteen patients were also analysed by a second radiologist with a similar experience level as that of the first. Nine measurements for IP, 13 measurements for IA, 11 measurements for EP, and 15 measurements for EA were performed. The correlation coefficients between the two sets of measurements were $0.46,0.30,0.67$, and 0.51 , respectively. There was no difference in the SWE values with respect to age, parity, and gravidity for any of the regions. The SWE values at the IA, IP, and EA regions between the postmenopausal and premenopausal women were significantly different $(p=0.038, p=0.059, p=0.065)$.

Conclusions: The posterior portion of the internal os is most likely to undergo inaccurate SWE measurement among the different anatomical positions. The correlation between radiologists was found to be different for different locations in the cervix. More studies are needed to determine the SWE values of the healthy cervix and the agreement levels between radiologists.

Key words: cervix, elastography, shear wave, transvaginal ultrasound.

\section{Introduction}

Shear wave elastography (SWE) is a new technique for measuring tissue elasticity, which depends on pressure that causes deformation of the tissue. The system uses acoustic radiation to induce mechanical vibrations and quantifies the stiffness by capturing the propagating shear waves [1]. The obtained data are analysed and used to create an elasto-

Correspondence address:

Dr. Aysegul Akdogan Gemici, Bakirkoy Dr. Sadi Konuk Training nd Research Hospital, Zuhuratbaba Mh. Tevfik Sağlam Cd. No: 11 Bakırköy, 34147, İstanbul, Turkey, e-mail: aysegulakdogan@yahoo.com

Authors' contribution:

A Study design · B Data collection · C Statistical analysis · D Data interpretation · E Manuscript preparation · F Literature search · G Funds collection 
graphic map. Different elasticity values are marked with different colours. The technique quantitatively measures tissue elasticity in kilopascals $(\mathrm{kPa})$ or metres per second $(\mathrm{m} / \mathrm{s})[1,2]$.

SWE can be used for a variety of solid organs, mainly thyroid and breast $[3,4]$. Recent literature has explored the use of SWE to evaluate gynaecological pathologies. Initially, strain elastography was used for elasticity assessment in earlier studies. However, as experience with the technique increased, SWE started to be preferred because of the advantages of the system, as it is almost unaffected by the depth of the cervix, and the generation of the mechanical vibration is not operator dependent [5-7]. SWE has been used mostly for assessing cervical insufficiency, premature delivery, and post-term pregnancy [8-11] in the gynaecological era. Recently, it has also been used for cervical tissue characterisation and the differentiation of benign and malignant cervical tumours [12,13].

To define and detect pathological changes rapidly and reliably, it is necessary to recognise the variability in the analysis of the healthy cervix. However, there are only a few studies about SWE analysis of the healthy, nonpregnant cervix $[14,15]$; consequently, there is a gap in the literature regarding changes in elasticity induced by patient-related factors and the interobserver variability of SWE measurements. Therefore, in this study, we aimed to analyse the SWE values of the healthy, nonpregnant cervix with respect to age, menopausal status, gravida, parity, and menstrual cycle by using the Toshiba SWE system at our institution.

\section{Material and methods}

Thirty-three patients who had given written, informed consent for US examination were enrolled in the present study, which had been rewieved appropriate by the Ethics Committee and was performed in accordance with the ethical standards described in an appropriate version of the 1975 Declaration of Helsinki, as revised in 2000. None of the patients had a history of cervical conisation or other conditions affecting the cervix. Patients with vertically oriented cervical canals were excluded. Other forms of the cervical canal (horizontal or angled) were analysed. The patients underwent transvaginal B-mode US examination followed by two-dimensional SWE in the same setting with an Aplio500 machine (Toshiba Medical System Corporation, Otawara, Tochigi, Japan). The transducer used for the grayscale US and SWE had a frequency range of 3 to $12 \mathrm{MHz}$.

For 15 patients, a second radiologist with the same experience level also performed SWE to determine the interobserver agreement level.

The women were asked to empty their bladders before the examination, where they were placed in the dorsal lithotomy position. The transducer was covered with a lubricated condom and placed in the anterior fornix of the vagina, and a sagittal view of the cervix, with the echogenic endocervical mucosa along the length of the canal, was obtained. The transducer was held gently, taking care to avoid any external compression during the acquisition. Elastograms were obtained at the internal and external os in the anterior (IA, EA) and posterior (IP, EP) portions of the cervix. Relatively homogenous regions in the tissue, away from boundaries and structures (e.g. blood vessels or Nabothian cysts) that could disrupt wave propagation, were chosen.

The shear wave frequency of the device was set to $4 \mathrm{MHz}$, and the tracking was set to 0 . This setting utilises a $4 \mathrm{MHz}$ push and $4 \mathrm{MHz}$ tracking pulse. It was possible to select a continuous or one-shot scan; we selected the one-shot scan setting to obtain higher image quality. During the five seconds required to stabilise the SWE, the images were captured and saved. The SWE images could be viewed using three different display modes after freezing: speed (shear velocity) mode (metres per second), elasticity mode (kilopascals), and propagation (arrival time contour) mode. Tissue stiffness-elasticity measurements were performed using a colour-coded map in the region of interest (ROI), representing the Young's modulus in metres per second at each pixel. SWE calculations were performed in the colour elastogram using a circular region of interest of $5 \mathrm{~mm}$ diameter. Tissue elasticity was displayed with a colour range from dark blue (lowest stiffness) to red (highest stiffness). The system displayed the mean elasticity values (E-mean) in metres per second. Measurements with standard deviations over $20 \%$ and patients presenting with heterogeneous regions or loss of colour - meaning a lack of technical conditions for real measurement - were excluded from the study. These values were measured three times, and the average value was taken. The total examination time was between 15 and $20 \mathrm{~min}$.

\section{Statistical analysis}

Data analysis was performed using SPSS version 26.0 (IBM Corporation, Armonk, NY). Descriptive data are presented as the means \pm SDs. A paired-samples $t$-test was used to compare the speed measurements obtained from each region of the cervix. The inter-operator agreement, which was calculated to compare the speed obtained from each operator, was determined using the intraclass correlation coefficient (ICC). Statistical significance was defined as a $p$ value less than 0.10 , due to the small number of patients.

\section{Results}

A total of 33 women were recruited for the study. The age range was from 27 to 63 years, with a mean age of 34 years. Thirteen patients were $\leq 40$ years old, and the remaining 20 patients were $>40$ years old. There were eight postmenopausal and 25 premenopausal women. Of the 25 premenopausal women, nine were in the secretory phase and 15 were in the proliferative phase. 
Table 1. Summary of mean speed obtained in each region as a function of patient characteristics

\begin{tabular}{|l|c|c|c|c|c|}
\hline Patient characteristics & $\begin{array}{c}\text { Number of } \\
\text { participants }\end{array}$ & $\begin{array}{c}\text { External os anterior } \\
\mathrm{m} / \mathbf{s}(\mathrm{SD})\end{array}$ & $\begin{array}{c}\text { External os posterior } \\
\mathrm{m} / \mathrm{s}(\mathrm{SD})\end{array}$ & $\begin{array}{c}\text { Internal os anterior } \\
\mathrm{m} / \mathrm{s}(\mathrm{SD})\end{array}$ & $\begin{array}{c}\text { Internal os posterior } \\
\mathrm{m} / \mathbf{s}(\text { SD) }\end{array}$ \\
\hline Age $\leq 40$ & 13 & $3.14(0.48)$ & $3.18(0.57)$ & $3.28(0.83)$ & $3.23(0.98)$ \\
\hline Age $>40$ & 20 & $3.19(0.51)$ & $3.20(0.62)$ & $3.44(1.01)$ & $3.66(1.01)$ \\
\hline Pre-menopause & 25 & $3.12(0.50)$ & $3.14(0.56)$ & $3.30(0.71)$ & $3.50(0.75)$ \\
\hline Post-menopause & 8 & $3.19(0.42)$ & $3.61(0.84)$ & $3.80(1.03)$ & $3.98(0.98)$ \\
\hline Follicular phase & 9 & $3.20(0.42)$ & $3.22(0.89)$ & $3.38(0.80)$ & $3.52(0.95)$ \\
\hline Luteal phase & 15 & $3.16(0.54)$ & $3.18(0.56)$ & $3.39(0.72)$ & $3.53(0.86)$ \\
\hline Nulliparous & 7 & $3.19(0.42)$ & $3.61(0.86)$ & $3.80(1.04)$ & $3.91(0.96)$ \\
\hline Multiparous & 26 & $3.10(0.50)$ & $3.12(0.59)$ & $3.3(0.72)$ & $3.50(0.80)$ \\
\hline
\end{tabular}

After excluding measurements with standard deviations over $20 \%, 33$ measurements from the IA, 26 measurements from the IP, 33 measurements from the EA, and 32 measurements from the EP of the cervix were obtained. The mean SWE values were $3.38 \pm 0.73,3.53$ $\pm 0.81,3.17 \pm 0.85$, and $3.18 \pm 0.84 \mathrm{~m} / \mathrm{s}$, respectively. The difference in speed among all regions was statistically significant $(p<0.05)$. A high positive correlation was found between IP and IA, EP and EA with Spearman's rho values of 0.70 and 0.61 . SWE values in each region in terms of the patient characteristics are summarised in Table 1.

Fifteen patients were also analysed by a second radiologist with a similar experience level as the first. Nine measurements for IP, 13 measurements for IA, 11 measurements for EP, and 15 measurements for EA were analysed. The correlation coefficients between the two sets of measurements were $0.46,0.30,0.67$, and 0.51 , respectively.

There was no difference in SWE values with respect to age, parity, and gravida for any of the regions. The SWE values of the IA, IP, and EA cervical regions between the postmenopausal and premenopausal women were significantly different $(p=0.038, p=0.059, p=0.065)$. The SWE values of the EP region did not differ between the two groups.

\section{Discussion}

Because of the spatial differences in the cervical tissue microstructure and variations in anatomical positioning, accurate usage of SWE is challenging [16,17]. While the external os can be evaluated easily, transducer angulation may be required after positioning gently onto the anterior fornix to analyse the internal os; nevertheless, shear wave propagation might be lost. Our results showed that a more significant proportion (seven patients, $21.2 \%$ ) of unsuccessful SWE measurements were made at the posterior part of the internal os. The anterior cervix produced more precise shear wave values than did the posterior cervix. Similar to the results of our study, O'Hara et al. found that the internal os in the posterior region of the cervix is most likely to produce inaccurate, or a loss of, shear wave propagation. They found that posterior angulated or vertically positioned cervixes are the most challenging anatomic positions for SWE analysis. In those cases, the posterior portion of the internal os can reach a depth greater than $3 \mathrm{~cm}$ from the transducer, a distance that shear waves cannot traverse [14].

Additionally, the cervix has high acoustic attenuation because of its microstructural complexity, which decreases the penetration depth of the main propagation pulse and reduces its ability to measure tissue displacement [16].

In most studies concerning the cervix, SWE yielded greater elasticity values in the posterior than in the anterior regions [16,18,19]. Edgar et al. hypothesised that depth was the reason for this difference but concluded that it was not significantly associated with SWE estimations; they stated that, instead, the differences might be related to tissue characteristics [20]. While we obtained SWE data on the sagittal axis of the uterine cervix, similarly to the literature $[5,21,22]$, they used cross-sectional views of the internal and external cervical os for measurements to analyse the effect of depth. By measuring the distance from the US transducer to different regions of interest for the same area of the cervix, they did not find any meaningful effect of depth in the SWE measurements [20].

In the present study, the obtained mean SWE values were similar to those in the literature [12-15]. The elasticity values were higher in the internal and posterior regions than in the external and anterior regions. In the study by Carlson et al. a gradient of elasticity was defined from the internal to the external os level, which was more conspicuous along the posterior than the anterior part of the cervix [16].

Our mean values from the four different locations also resembled the aforementioned gradient. In addition, a high positive correlation was found in the elasticity values between the IP and IA regions and between the EP and EA regions of the cervix, as was first described in the literature.

Unlike older, more challenging elastography techniques such as strain elastography imaging, SWE pro- 
duces mechanical impulses by itself, and with the help of this self-produced impulse, different groups have demonstrated improved reproducibility $[2,14,18]$. However, the pressure on the cervix, generated during the positioning of the probe at the anterior fornix, might be unavoidable, which may alter the SWE measurements. O'Hara et al. concluded that increasing the transducer pressure also caused the SD of the mean speed to increase [14]. Consequently, as mentioned above, here only a small amount of pressure was applied when the probe made contact. It was not our aim to investigate the effect of probe compression; we mainly aimed to determine the consistency of cervix SWE measurements and changes resulting from patient-related factors. The magnitudes of the correlation coefficients were low to medium for the internal os and medium to high for the external os. Our interobserver agreement levels were lower than those from the Ohara et al. study, which was the only one to analyse the interobserver agreement of healthy cervix SWE measurements that produced high levels for all regions [14].

We also note that although the number of measurements in the posterior part of the cervix was low, the compatibility was greater from the anterior parts. It is believed that the microstructure of the uterine cervix might be the reason for these results.

In the study by Ohara et al., which used the same Toshiba system as in our study, the authors concluded that there was an overall increase in cervical stiffness with aging, but they did not mention any differences that could be attributed to menopausal changes. In the present study, age was not a factor in the difference in SWE estimations. However, menopausal status was related to the stiffness of the cervix; postmenopausal patients had higher SWE values than premenopausal patients at the internal os. Thomas et al., who were the first to compare the values of real-time cervix sonoelastography between a healthy group and patients with cervical lesions in the literature in 2007, found no difference in elasticity between healthy pre- and postmenopausal women [13]. Although we concluded that postmenopausal hormonal changes of the cervix might alter the SWE values, we observed no significant difference in the variability of the elasticity indices for the different menstrual phases. The study from Manchanda et al., which was the first in the literature to describe SWE of the healthy cervix, demonstrated no effect of the menstrual cycle [15]. However, O'Hara et al. found that the SWE speed at the internal and external os may also differ at different stages of the menstrual cycle [14]. The greatest speeds were obtained at the internal os during the luteal phase of ovulation, and before menstruation the cervix becomes softer. In the present study, the low number of studied premenopausal women might be the reason for our discordant results.

\section{Study limitations}

The small sample size and the study being conducted in a single centre were the main limitations.

\section{Conclusions}

Accurate SWE measurements are challenging because of the cervical anatomy and microstructure. Accordingly, consistency is an important issue, but it was low in the present study. Analysing the changes in the elasticity values of the healthy cervix resulting from patient-related factors in different conditions might be helpful for obtaining basal reference data.

\section{Conflict of interest}

The authors report no conflict of interest.

\section{References}

1. Barr RG. Real-time ultrasound elasticity of the breast. Ultrasound Q 2010; 26: 61-66.

2. Bamber J, Cosgrove D, Dietrich CF, et al. EFSUMB guidelines and recommendations on the clinical use of ultrasound elastography. Part 1: basicprinciples and technology. Ultraschall Med 2013; 34: 169-184.

3. Gong X, Xu Q, Xu Z, et al. Real-time elastography for the differentiation of benign and malignant breast lesions: a meta-analysis. Breast Cancer Res Treat 2011; 130: 11-18.

4. Ding J, Cheng H, Ning C, et al. Quantitative measurement for thyroid cancer characterization based on elastography. J Ultrasound Med 2011; 30: 1259-1266.

5. Swiatkowska-Freund M, Preis K. Elastography of the uterine cervix: implications for success of induction of labor. Ultrasound Obstet Gynecol 2011; 38: 52-56.

6. Tekesin I, Wallwiener D, Schmidt S. The value of quantitative ultrasound tissue characterization of the cervix and rapid fetal fibronectin in predicting preterm delivery. J Perinat Med 2005; 33: 383-391.

7. Thomas A. Imaging of the cervix using sonoelastography. Ultrasound Obstet Gynecol 2006; 28: 356-357.

8. Agarwal A, Agarwal S, Chandak S. Role of acoustic radiation force impulse and shear wave velocity in prediction of preterm birth: a prospective study. Acta Radiol 2018; 59: 755-762.

9. Agarwal S, Agarwal A, Joon P, Saraswat S, Chandak S. Fetal adrenal gland biometry and cervical elastography as predictors of preterm birth: a comparative study. Ultrasound 2018; 26: 54-62.

10. Ono T, Katsura D, Yamada K, et al. Use of ultrasound shear-wave elastography to evaluate change in cervical stiffness during pregnancy. J Obstet Gynaecol Res 2017; 43: 1405-1410.

11. Carlson LC, Romero ST, Palmeri ML, et al. Changes in shear wave speed pre-and post-induction of labor: a feasibility study. Ultrasound Obstet Gynecol 2015; 46: 93-98. 
12. Liu C, Li TT, Hu Z, et al. Shear wave elastography in the diagnosis of cervical disease. J Ultrasound Med 2019; 38: 3173-3181.

13. Thomas A, Kümmel S, Gemeinhardt O, Fischer T. Real-time sonoelastography of the cervix: tissue elasticity of the normal and abnormal cervix. Acad Radiol 2007; 14: 193-200.

14. O’Hara S, Zelesco M, Sun Z. Shear wave elastography on the uterine cervix: technical development for the transvaginal approach. J Ultrasound Med 2019; 38: 1049-1060.

15. Manchanda S, Vora Z, Sharma R, et al. Quantitative sonoelastographic assessment of the normal uterus using shear wave elastography: an initial experience. J Ultrasound Med 2019; 38: 3183-3189.

16. Carlson LC, Feltovich H, Palmeri ML, et al. Estimation of shear wave speed in the human uterine cervix. Ultrasound Obstet Gynecol 2014; 43: 452-458.

17. Feltovich H, Carlson L. New techniques in evaluation of the cervix. Semin Perinatol 2017; 41: 477-484.
18. Hernandez-Andrade E, Hassan SS, Ahn H, et al. Evaluation of cervical stiffness during pregnancy using semiquantitative ultrasound elastography. Ultrasound Obstet Gynecol 2013; 41:152-161.

19. Peralta L, Molina FS, Melchor J, et al. Transient elastography to assess the cervical ripening during pregnancy: a preliminary study. Ultraschall Med 2017; 38: 395-402.

20. Hernandez-Andrade E, Aurioles-Garibay A, Garcia M, Korzeniewski SJ, et al. Effect of depth on shear-wave elastography estimated in the internal and external cervical os during pregnancy. J Perinat Med 2014; 42: 549-557.

21. Fruscalzo A, Steinhard J, Londero AP, et al. Reliability of quantitative elastographyof the uterine cervix in at-term pregnancies. J Perinat Med 2013; 41: 421-427.

22. Molina F, Gomez L, Florido J, et al. Quantificationof cervical elastography. A reproducibility study. Ultrasound Obstet Gynecol 2012; 39: 685-689. 